\title{
Envejecimiento y trato hacia las personas mayores en Chile: una ruta de la desigualdad persistente
}

\author{
Aging and treatment to the elderly in Chile: \\ A Path of persistent inequality \\ Diosnara Ortega GonzÁlez* \\ "Académica Escuela Sociología Universidad Católica Silva Henríquez \\ $\bowtie$ dortegag@ucsh.cl
}

\begin{abstract}
RESUMEN
El envejecimiento en Chile está atravesado por desigualdades múltiples, las que se potencian bajo la condición de ser adulto mayor. La vejez remite ella misma a una producción de desigualdad categorial expresada entre otras formas, en el trato que reciben las personas mayores por parte de otros actores e instituciones. El artículo ahonda en las rutas de producción de la desigualdad persistente específicamente en grupos de adultos mayores y su relación con el maltrato. Los resultados presentados se sustentan en la Encuesta de Percepción del Trato hacia Adultos Mayores realizada en las comunas de Recoleta y Las Condes a una muestra de 198 adultos mayores durante 2017. Ellos permiten evidenciar la presencia de diferentes expresiones de maltrato hacia estos adultos mayores, fundamentalmente de tipo psicológico, donde la condición de género y vejez junto a otras como la convivencia con familiares, el nivel socioeconómico y educacional amplifican la reproducción de desigualdades persistentes.
\end{abstract}

PALABRAS CLAVE: Envejecimiento, Chile, desigualdad persistente, adultos mayores, maltrato.

\begin{abstract}
Aging in Chile is crossed by multiple inequalities, which are enhanced under the condition of being an adult. Old age itself refers to a production of categorical inequality expressed, among other forms, in the treatment that older people receive from other actors and institutions. The article delves into the production routes of persistent inequality


specifically in groups of older adults. The results presented are based on the Survey on the Perception of Treatment of Older Adults carried out in the communities of Recoleta and Las Condes with a sample of 198 older adults during 2017. They make it possible to demonstrate the presence of different expressions of abuse towards these older adults, fundamentally of a psychological nature, where the condition of gender and old age together with others such as living with family members, the socioeconomic and educational level amplify the reproduction of persistent inequalities and associated practices.

KEY WORDS: Aging, Chile, persistent inequality, older adults, abuse.

\section{INTRODUCCIÓN}

La realidad del envejecimiento poblacional creciente y acelerado que vive la sociedad chilena, ha sido una problemática que ha tomado centro tanto en la agenda de las ciencias sociales como en las políticas públicas del país.

La transición demográfica presente en Chile desde 1975 y que se acentúa a partir del año 2000, ha implicado un ritmo de crecimiento de la población adulta mayor al doble en relación al promedio nacional y al de los menores de 60 años (Huenchuán, et.al, 2007). Chile se encuentra, junto a Cuba y Uruguay, entre los países con un proceso de envejecimiento más acelerado, proyectando hacia el 2025 más de un 20\% de adultos mayores en su población (CEPAL, 2009).

Si bien este hecho nos muestra una realidad social-y no solo demográfica-, las inquietudes que siguen los estudios y las políticas al respecto se encaminan no solo a preguntarse por cuánto envejece Chile, a qué ritmo, sino también cómo se envejece. Las investigaciones al respecto han venido mostrando que tan preocupante es el proceso de envejecimiento constante y acelerado, como su multidimensionalidad (Huenchuán, 2007, 2009; Thumala, 2011; Guajardo, 2013; Abusleme, 2014). La multiplicidad de procesos y dimensiones sociales que atraviesan el envejecer y a las que el envejecimiento afecta, dan cuenta de una situación que nos convoca e involucra como sociedad.

Rasgos y procesos como la feminización del envejecimiento, la violencia y el maltrato, la vulneración de los derechos, la fragilidad de los cuidados, son parte del envejecimiento en Chile (Gonzálvez, 2017; Del Pozo, 2014; Briceño, et.al, 2014; González, 2014). Ahora bien, ¿son estos procesos aislados unos de otros o constituyen (pueden ser leídos) desde una lógica estructural de (re)producción de la desigualdad persistente? Partiendo de la tesis de Charles Tilly (2000) sobre la institucionalización de pares categoriales por medio de los cuales se produce la desigualdad persistente, entendemos que las categorías de género y edad constituyen dos sistemas mediante los cuales pueden explicarse la reproducción de la desigualdad persistente en el proceso de envejecimiento de los adultos mayores en Chile. El trato que reciben estos grupos 
constituye una expresión última por medio de la cual podemos rastrear las huellas de un proceso de desigualdad persistente. En este sentido proponemos un acercamiento a una forma específica de reproducción de las desigualdades que es el maltrato, a la vez que consideramos es el mismo no solo reproductor de desigualdades sino la expresión de desigualdades ya instaladas, como las de género y etarias.

El objetivo del artículo es mostrar a partir de los resultados de la Encuesta de Percepción del Trato hacia los Adultos Mayores (EPTAM), realizada en las comunas de Recoleta y Las Condes a una muestra de 198 adultos mayores ${ }^{1}$ durante los meses de mayo-julio del 2017, la presencia de una desigualdad persistente común adscrita a la condición de la vejez y género más allá de las desigualdades particulares (nivel socioeconómico, nivel educacional, territorio), y cómo estas están asociadas a experiencias de maltrato de diverso tipo que viven estos sujetos.

Nuestra hipótesis de trabajo ha sido que si bien las desigualdades de clase atraviesan los modos en que se envejece en Chile, la vejez constituye ella misma una categoría productora de desigualdad persistente que permea otras desigualdades, expresándose y reproduciéndose mediante diversas formas de maltrato.

La sola condición de envejecer remite a la activación de un nuevo tipo de desigualdad que es claramente potenciada con la presencia de otras múltiples: de género, clase, etnia. Estas formas de desigualdades, todas, implican la reproducción de maltrato, son su contexto de posibilidad. En síntesis, proponemos comprender la desigualdad como expresión y condición de diversas formas de maltrato. La desigualdad conlleva potencialmente al maltrato, a su vez las diversas formas de maltrato expresan jerarquías de desigualdad instaladas en los imaginarios y prácticas de quienes las reproducen.

En este artículo se presentan los resultados más significativos de la EPTAM, con el énfasis de mostrar las percepciones en torno al trato que reciben las personas mayores y cómo este devela la trama de una desigualdad persistente.

\section{El maltrato hacia las personas mayores: una realidad invisibilizada}

Los estudios nucleados alrededor del tema maltrato parten de una realidad, las diversas y numerosas expresiones de maltrato hacia adultos mayores, así como su ocultamiento e invisibilización. El reconocimiento del maltrato hacia otros grupos vulnerables como los niños/as y las mujeres, cuenta en la sociedad chilena con una mayor representación en términos de imaginarios que el maltrato hacia las personas mayores (Abusleme,2013). Algunas investigaciones muestran una significativa presencia del maltrato hacia este grupo, especialmente de tipo psicológico (Caballero \& Massad, 2013; Abusleme y Caballero, 2013), sin embargo no siempre es percibido o reconocido como tal socialmente, incluyendo a los propios adultos mayores.

La EPTAM estuvo organizada por la Escuela de Sociología de la Universidad Católica Silva Henríquez en colaboración con el SENAMA. Se aplicó en las comunas de Recoleta y Las Condes durante 2017. Ver apartado metodológico. 
A diferencia de lo que ocurre a nivel de imaginarios, los avances en materia jurídica-normativa han tenido un desarrollo en este ámbito en el país. En 2005 la Mesa de Trabajo sobre Maltrato al Adulto Mayor, definió y tipificó el Maltrato al adulto mayor. En este documento el mismo se define como: la acción u omisión que produce daño a un adulto mayor y que vulnera el respeto a su dignidad y el ejercicio de sus derechos como persona, pudiendo realizarse de manera intencionada o por desconocimiento, de manera no intencionada. El daño producido a un adulto mayor puede ser de orden físico, psíquico y/o patrimonial (SENAMA, 2005).

Los tipos de maltratos reconocidos en este documento son:

Maltrato físico: Uso de la fuerza física en contra de un adulto mayor que daña su integridad corporal, puede provocar dolor, lesión y/o discapacidad temporal o permanente, y en casos extremos, la muerte.

Maltrato psicológico: Acciones que producen angustia, pena, estrés, sentimientos de inseguridad, baja autoestima, y/o agreden la identidad, dignidad y respeto de la autonomía de una persona mayor.

Abuso sexual: Cualquier acción de carácter, significación o connotación sexual con una persona mayor sin su consentimiento, empleando la fuerza, amenaza y/o engaño, aprovechándose de su deterioro físico o psíquico.

Abuso patrimonial: Mal uso, explotación o apropiación de los bienes de la persona mayor por parte de terceros, sin consentimiento o con consentimiento viciado, fraude o estafa, engaño o robo de su dinero o patrimonio.

Negligencia: Es el incumplimiento por deserción o fracaso de las funciones propias del cuidado para satisfacer las necesidades vitales de una persona mayor (higiene, vestuario, administración de medicamentos, cuidados médicos). La negligencia puede ser activa o pasiva (...)

Abandono: Se produce cuando cualquier persona o institución no asume la responsabilidad que le corresponde en el cuidado del adulto mayor, o que habiendo asumido el cuidado o custodia de un adulto mayor lo desampara de manera voluntaria

Maltrato estructural o societario: Aquel que ocurre desde y en las estructuras de la sociedad mediante normas legales, sociales, culturales, económicas que actúan como trasfondo de todas las otras formas de maltrato existente (SENAMA, 2005). 
Uno de los factores que influyen en la producción del maltrato, en todas sus expresiones y no solo el estructural, son las percepciones negativas en torno a la vejez (Guajardo, 2013; Caballero, Massad, 2013).La marginación estructural a la que son sometidas las personas mayores tienen en su base una interpretación de esta etapa de la vida en términos de dependencias, desvalorización. Sin considerarse que probablemente una de las pérdidas fundamentales impuestas socialmente a las personas mayores es precisamente el de su valor como sujetos productivos en toda su dimensión y no solo económica. Esta es una de las principales formas de expropiación a las que como Sociedad, Estado, Mercado, Familia, sometemos consciente o inconscientemente a las personas mayores. Este solo proceso de expropiación constituye en sí mismo un acto de maltrato y también de producción de desigualdad.

Los elementos hasta acá señalados permiten mostrar cuatro tesis sobre las cuales se estructuró la EPTAM y que retomamos en este artículo: (1) el maltrato no es solo una cuestión individual o intrafamiliar, sino que obedece a condiciones estructurales, de relaciones, imaginarios sociales mediante las cuales se ordena la vida en sociedad. (2) El maltrato no afecta solo a quienes lo vivencian en su condición de personas mayores víctimas por tal condición, sino también a otros grupos sociales encargados directa o indirectamente de su bienestar, ya sea por acción directa u omisión de sus responsabilidades respecto de los adultos mayores. (3) Las expresiones del maltrato son múltiples - tal cual reconoce su tipificación (SENAMA, 2005)- y obedecen a causas y condiciones múltiples. (4) El maltrato es una expresión de las desigualdades persistentes a la vez que estas desigualdades potencian la producción del maltrato.

La producción de desigualdades es una condición de los modos en que se vive la vejez en Chile y a la vez es el envejecimiento junto a otras desigualdades como las de género, detonantes de malos tratos, exclusiones y vulneraciones que contextualizan la vida de estos adultos mayores.

\section{Desigualdad persistente}

La noción de desigualdad persistente remite, según Charles Tilly a la presencia de determinadas categorías sociales que funcionan como marcadores sociales muchas veces representados como biológicos (joven/viejo, hombre/mujer), cuando en realidad estas categorías dependen no de factores "naturales" sino de una determinada organización social, de creencias e imposiciones sociales extensivas (Tilly, 2000, p.21). Esta desigualdad persistente es "efectiva" a un determinado sistema de ordenamiento social, contribuye ella a un determinado modo de producción y distribución (desigual) de los recursos, en rigor, constituye su justificación. Estos sistemas categoriales en rigor lo que establecen son sistemas de cierre de clase, exclusión y control sociales (Tilly, 2000, p. 21).

En ese sentido las desigualdades producidas por medio de los principios diferenciadores de raza, género, etnia, clase, edad, ciudadanía, nivel educacional entre otros, funcionan por

medio de sistemas de categorías en donde no solo se clasifica a los sujetos, sino que se les distribuye socialmente en un sistema de producción de desigualdades. 
Según Tilly (2000):

los seres humanos inventaron la desigualdad categorial hace miles de años y la aplicaron a una amplia gama de situaciones sociales. Aunque sea inadvertidamente, la gente establece sistemas de desigualdad categorial fundamentalmente por medio de estos dos mecanismos causales: (1) la explotación y (2) el acaparamiento de oportunidades (...). (p.23)

Estudios sobre maltrato hacia personas mayores en Chile dan cuenta de cómo son producidos como sujetos "improductivos", "dependientes", a los que se les desvaloriza no solo por medio de creencias e imaginarios sino por medio de un sistema de organización de de normativas, recursos y derechos (Huenchuán, 2014; Abusleme y Caballero, 2014).

Los adultos mayores constituyen una parte significativa del ejército de reserva en sociedades donde la brecha entre sistema de seguridad social, salario y consumo es tal que arroja a las personas a reinsertarse en el mercado laboral una vez alcanzada su edad de jubilación. Según encuesta CASEN, 2013, el promedio de pensión en Chile es de \$137000. Con estas pensiones, el aumento de la esperanza de vida que alarga en más de 20 años la vida pos-jubilación, y un $60 \%$ menos de ingresos como promedio de lo que se ganaba en su edad económicamente activa (Valenzuela, 2016), la realidad estructural de las personas mayores se constituye desde una producción profundamente desigual respecto de las personas jóvenes.

Las condiciones de dependencia económica de las personas mayores no constituyen solamenteuna expresión de la pauperización dela vejez sino antes bien cómo operan los marcadores sociales (joven/viejo) en la producción de una desigualdad persistente. Esta desigualdad, como toda desigualdad, pauperiza a unos (las personas mayores) mientras es efectiva a otros (aquellos que explotan o acaparan el trabajo desvalorizado de las personas mayores).

Tal cual evidencia el Informe Mercado Laboral, Adulto Mayor y Personas próximas a jubilar en Chile, del SENAMA (2016), las bajas pensiones llevan a que la población próxima a jubilar intente permanecer insertada laboralmente o retorne una vez jubilada, esto a pesar de que las remuneraciones disminuyen a partir de los 60 años, especialmente a partir de los 75 y las condiciones laborales se vuelven mucho más flexibles, pasando de ocupado tradicional a ocupado sin remuneración tradicional, principalmente en el caso de las mujeres mayores de 60 años (SENAMA, 2016, p. 22) ¿Qué significa que las personas mayores se reinserten en el mercado laboral en estas condiciones, y aún más que significa que se reinserten? El cierre social opera visiblemente, al extremo que estas personas muchas veces continúan empleados en sus mismos espacios laborales, solo que en nuevas condiciones laborales donde se flexibiliza ostensiblemente su empleo por la sola razón de "entrar" dentro de un rango etario.

La percepción social negativa respecto a la vejez, en donde esta es entendida como "carga" a la familia y la sociedad misma, termina no solo produciendo maltrato estructural 
sino legitimando la expulsión de este grupo del mercado laboral o bien flexibilizando su empleo, es decir produciendo desigualdad categorial. La subcontratación y flexibilización de mano de obra de adultos mayores, así como el no reconocimiento de las labores de cuidado que realizan dentro de la familia, generan una "falsa" dependencia de este grupo respecto de la mano de obra económicamente activa, lo cual en el fondo termina siendo rentable al capital y el mercado. Esto es, los procesos de acumulación y explotación como mecanismos de producción de la desigualdad categorial (Tilly, 2000, p. 22-24).

Las situaciones de dependencia, tanto física, emocional-psicológica, como económicas, han demostrado ser una de las causas intervinientes en la presencia de violencia y maltratos, su "naturalización" y/o reproducción. A la vez la dependencia económica es expresión y/o potencia una relación de desigualdad estructural, entre el dependiente y quien controla los recursos y los distribuye.

La presencia de la desigualdad persistente, que opera mediante el principio diferenciador de la edad, conjugado con otros como el de género, son la base constitutiva en nuestro criterio de la producción del maltrato hacia las personas mayores. El maltrato es su expresión más directa, y este se reproduce por medio de un sistema organizacional que es la desigualdad persistente, la que opera a nivel estructural y de nuestras prácticas.

\section{Aspectos metodológicos}

Los resultados presentados se sostienen sobre la Encuesta de Percepción del Trato hacia Adultos Mayores realizada en 2017 en las comunas de Recoleta y Las Condes, de la Región Metropolitana de Santiago. El objetivo de la misma fue revelar las percepciones que tienen los propios adultos mayores respecto del trato que reciben en distintos espacios y por diferentes actores (Familia, Estado, Mercado), considerando las desigualdades que atraviesa la vejez en Chile, y también el maltrato.

La EPTAM tuvo lugar como una actividad de aprendizaje y servicio desarrollada entre el Programa Buen Trato al Adulto Mayor del SENAMA y la Escuela de Sociología de la Universidad Católica Silva Henríquez, bajo la coordinación de la autora.

La muestra seleccionada estuvo dirigida bajo dos requisitos previos: (1) la selección de comunas diversas y desiguales entre sí, en donde se reprodujera la desigualdad territorial; y (2) el acceso expedito a los adultos mayores considerando los recursos tiempo y económicos. En función de ello y en acuerdo con los/as encargados del Programa en las comunas, se decidió escoger una muestra intencionada de adultos mayores asistentes a instancias que desarrolla SENAMA en los territorios, tales como Casas del Adulto Mayor, Centros Diurnos y Círculos de Encuentro del Adulto Mayor.

En la comuna de Recoleta donde se aplicaron 98 encuestas, las mismas se distribuyeron entre el Centro Diurno del Adulto Mayor, y la Casa del Adulto Mayor, mientras en Las Condes las 100 encuestas realizadas fueron entre los Círculos de Encuentro del adulto mayor Rosita 
O’ Higgins, Los Dominicos y El Canelo. Al respecto deben señalarse varios aspectos relevantes en la interpretación de los resultados acá presentados, los cuales deben considerase así mismo como limitaciones del estudio:

1- La población que asiste a estos centros convoca a adultos mayores con diferentes necesidades, realidades, y ofrece servicios diferenciados, que van desde adultos mayores en situación de mayor dependencia (física, intelectual, económica), hasta adultos mayores con necesidades de superación, socialización y sin situación de dependencia explícita.

2- Las diferencias de estos subgrupos al interior del grupo adultos mayores, se expresan al interior de las comunas y entre ellas. El tipo de instituciones que nuclea a los adultos mayores a los cuales se accedió en la comuna de Recoleta ofrece servicios más básicos de atención a su población mientras las instituciones de Las Condes, los Círculos de Encuentro, más que servicios básicos, representan espacios de socialización de sus asistentes con actividades más especializadas. Las diferencias de perfil entre unas y otras instancias de participación también median el tipo de adulto mayor encuestado.

3- La población asistente a estos espacios, con todas sus diferencias, forman parte de los programas dirigidos por SENAMA, los conocen y participan en ellos. Estos adultos mayores cuentan con redes que las mismas instituciones favorecen, se movilizan, con ayuda o solos hasta estos lugares, por lo cual pueden ser calificados como grupos con autonomía, independencia y empoderamiento, o en busca de ello, lo cual es ya en sí una expresión de aquello. Esta es una cualidad no menor si se piensa en un universo mayor con realidades muy distantes de adultos mayores que carecen de redes, apoyos institucionales, autonomía, entre otras condicionantes.

4- En estos espacios donde se desarrollan actividades destinadas a la socialización, fortalecimiento de habilidades manuales, intelectuales, entre otras, hay una sobrerrepresentación femenina, la cual también debe ser leída bajo la reproducción de un paradigma patriarcal donde se constituyen determinados patrones de lo femenino y lo masculino. Al ser espacios que efectivamente funcionan también como clasificadores sociales al estar orientados a un público específico (adultos mayores), se asocia igualmente como espacios para sujetos dependientes, desvalorizados, en los que "cabe" la mujer, no así el hombre.

La encuesta estructuró 19 preguntas, además de las referidas a datos sociodemográficos. El procesamiento de la información se realizó con SPSS. Las preguntas se ordenaron a partir de una operacionalización del maltrato, según tipificación del SENAMA (2005). La encuesta, de carácter anónimo, buscaba obtener información sobre experiencias y percepciones del trato hacia las personas mayores, desde las realidades 
personales de los/as encuestados/as. La misma fue aprobada por el Programa de Buen Trato al Adulto Mayor a nivel nacional y de las comunas según sus protocolos éticos. De igual manera fue certificada por el comité ético de la Escuela de Sociología de la UCSH desde donde se llevó a cabo la investigación.

Se dirigieron preguntas referidas no solo a conocer percepciones y experiencias respecto del Trato, sino también poder identificar los actores o instituciones principales desde donde se produce y cómo se produce ese trato, ya sea desde la Familia, el Estado, el Mercado, o la Comunidad. Se tomaron como variables no solo aquellas que expresan directamente tipos de maltratos, sino otras que consideramos posibles intervinientes en las vivencias del maltrato y sus percepciones, tales como variables socioeconómicas, sexo, niveles de dependencia, número de hijos, lugar de residencia, derechos entre otras. Este perfil permitió un acercamiento al maltrato, sus múltiples expresiones y desigualdades que lo producen/potencian.

\section{RESULTADOS}

Antes de presentar el análisis de los resultados más significativos de la EPTAM, es preciso reseñar algunos datos sociodemográficos de la muestra pertinentes para la posterior lectura. Respecto de la muestra total de 198 personas, debe señalarse que hubo una sobre representación de mujeres, para un total del 78,8\%. Este dato responde no solo al hecho de que la población femenina adulta mayor sea superior a la de los hombres, como tendencia demográfica, sino también a las características de la muestra seleccionada tal cual se explicó en el apartado metodológico. El promedio de edad fue de 75 años. Respecto del nivel educacional del total de la muestra un 40\% que declaró tener como última enseñanza terminada, la media, y un 34\% nivel universitario. Este dato cobra diferencias entre comunas. En Las Condes es superior la cantidad de encuestados/as universitarios/as (56\%), correspondiendo a la enseñanza media un 34\%. Mientras en Recoleta, el 47\% tiene como último nivel alcanzado la enseñanza media, un 37\% posee solo enseñanza básica y apenas un 11\% ha alcanzado nivel universitario. Esta es una de las expresiones de las desigualdades sociales "enclasadas" al interior de este grupo.

El hecho del alto nivel educacional encontrado entre las/os encuestadas/os en Las Condes, con más del 50\% de universitarios es un dato explicativo también de los niveles de independencia y autonomía con que cuentan estas personas, así como del rango de sus pensiones, tal cual se leerá más adelante. El indicador del nivel educacional da cuenta de una presencia de desigualdad persistente a nivel de clase, la cual se reproduce y afecta también otras, como la condición de vejez. Veamos entonces las principales variables por medio de las cuales pudimos sondear las condiciones en que se produce el trato hacia las personas mayores y en consecuencia la presencia de aquellas desigualdades que atraviesan la vejez y cómo esta potencia a su vez otras "nuevas" desigualdades. 
Dependencia económica y Abuso patrimonial

La dependencia económica de los/as adultos mayores encuestados/as no dice inmediatamente sobre la presencia de abuso patrimonial, pero sí coloca una alerta sobre un potencial para ello y a la vez dice de las condiciones de desigualdad en las que son insertos/ as. Un dato interesante desde donde leer estos resultados y especialmente los referidos a la autonomía en la administración de los bienes propios es el género. El 78,8\% de la muestra son mujeres. Las subvariables a partir de las cuales exploramos los niveles de dependencia económica y la presencia de abuso patrimonial, están todas mediadas por la condición de género que caracteriza la muestra, como se analiza a continuación. Las limitaciones metodológicas del estudio no permiten establecer una comparación entre géneros (sexo) pero si resalta la necesidad de analizar los datos acá presentados desde el género mismo.

\section{Aportes monetarios realizados por adultos mayores al grupo familiar}

Un dato contextual desde el cual leer tanto las condiciones de dependencia económica, así como la existencia de maltratos vividos o potenciales, es la presencia de otras personas con las cuales conviven o no las personas mayores. Los resultados de la encuesta arrojaron que el $71 \%$ de los/as encuestados/as vivía con familiares, un $25 \%$ solos y un $4 \%$ con otras personas no familiares. Este es un dato relevante para comprender en cabalidad las condiciones en las cuales podemos considerar la presencia de dependencia económica en los/as adultos mayores.

Del total de encuestados/as el 62\% realiza aportes monetarios al grupo familiar, no necesariamente al núcleo familiar con el cual viven. Este por ciento de aportes es mayor en aquellos grupos con mayores ingresos, como en el caso de Las Condes donde un 67\% declara realizar aportes monetarios, mientras que en Recoleta desciende al 57\%. En ambos casos es significativo que más de la mitad realiza aportes monetarios al grupo familiar.

\section{Pensiones y ayudas económicas}

Si nos adentramos en los valores de las pensiones y las ayudas económicas que estos/ as reciben y cómo se distribuyen al interior de las comunas, se observará una desigualdad persistente de clase que se expresa en los ingresos, así como antes se indicó en el nivel educacional. En Las Condes el 41\% recibe una pensión de 300. 000 pesos o más, mientras en Recoleta el 41\% percibe entre 101. 000 y 155.000 pesos. Apenas el 6\% en Recoleta gana como pensión 300.000 pesos o más. En esta comuna el 90\% recibe una pensión igual o inferior a 250. 000 pesos, mientras en Las Condes solo el $45 \%$.

Ahora bien, el monto de las pensiones guarda directa relación con el nivel educacional. Del total de encuestados/as, de los que reciben una pensión igual o superior a 300. 000 
pesos, poseen como último nivel alcanzado el universitario, el 15,7\%. Si se observa este dato desagregado, en Las Condes de quienes reciben 300. 000 pesos o más como pensión el 34\% poseen como último nivel alcanzado el universitario (29\%) o posgrados (5\%). En Recoleta en cambio el porciento de representación mayor se concentra en el tramo que recibe entre 101.000 y 155.000 pesos, de ellos teniendo como último nivel educacional alcanzado la enseñanza media $(21,4 \%)$ y la básica $(15,3 \%)$.

\section{Pensiones y aportes monetarios que realizan adultos mayores a la familia}

Para profundizar en el conocimiento de las desigualdades y su expresión se cruzaron las variables pensiones y aportes monetarios al grupo familiar. El resultado fue que del 62\% que declara hacer aportes a la familia regularmente, el 19\% se concentra en quienes ganan 300.000 pesos o más realizan aportes al grupo familiar. Mientras que el 12\% de quienes realizan aportes perciben pensiones entre 156.000 y 250. 000 pesos y un 11\% reciben entre 101.000 y 155.000 pesos.

En Las Condes el 33\% de quienes perciben 300.000 o más pesos como pensión realizan aportes económicos al grupo familiar, y en general en todos los tramos, excepto aquellos que reciben menos de 100.000 pesos. En Recoleta se concentran los aportes en el tramo donde a la vez se encuentran la mayoría de las pensiones recibidas, entre 101.000 y 155.000 pesos, pero con apenas un $17,3 \%$, siendo superior en este tramo la cifra de quienes no aportan, el 23,5\%. Los datos confirman dos situaciones: las desigualdades persistentes expresadas por medio de los ingresos, y a la vez paradójicamente la común participación de los adultos mayores en la economía familiar (67\% de los AM de Las Condes realiza aportes y el 57\% de los de Recoleta).

\section{Procedencia de las ayudas económicas}

Si bien los/as adultos mayores tienen una participación en los aportes realizados a la familia, es también de ella que se obtienen otros beneficios económicos adyacentes a las pensiones. El 62\% recibe aportes económicos provenientes de la Familia, mientras solo un 15\% del Estado. Los aportes recibidos por parte de la familia son significativamente superiores a los recibidos por el Estado en ambas comunas. Este dato muestra cómo la familia es para estos casos proveedor principal de recursos económicos, muy por encima del Estado u otros agentes. La urgencia de políticas públicas y un sistema de seguridad social que contribuya al apoyo también económico de nuestros adultos mayores, es un hecho. Ahora bien, allí donde se comprueban desigualdades de clase, expresadas mediante los capitales que poseen en una y otra comuna las personas mayores, la ausencia/presencia del Estado puede y suele estar atravesada también por dichas desigualdades. Del mismo modo allí donde las familias poseen menores recursos (económicos, de redes, psicológicos, etc.) se hace más 
vital la presencia del Estado, siendo que se confirma provienen de ella significativamente estos apoyos. Con ello no queremos decir que la lógica sería reforzar la presencia del Estado allí donde las redes familiares son más débiles, porque ello sigue manteniendo a la familia como el actor proveedor fundamental del bienestar y los cuidados, sino antes bien fortalecer la presencia del Estado como administrador del bien común en los apoyos que reciben los adultos mayores, de modo que no queden "sujetos" a la reproducción de desigualdades persistentes, como las de clase, las que con la vejez, se refuerza.

\section{Actividades que realizan al menos una vez a la semana los adultos mayores}

Además de los aportes monetarios, la segunda forma de contribución económica sobre la que indagamos fue la presencia de actividades con un valor de cambio (casi nunca reconocido como tal) que realizan muy frecuentemente las personas mayores. De las 4 labores consultadas ${ }^{2}$, el 90\% afirmó que al menos una vez a la semana realizan compras propias y de la familia, mientras el 85\% cocina o realiza labores domésticas. Hay que considerar acá el promedio de edad de estas personas que es de 75 años y el 78,8\% son mujeres. Resalta que con un promedio de edad de 75 años la actividad que menos realizan es trabajo remunerado, siendo este dato consistente con el Informe Mercado Laboral, Adulto Mayor y Personas próximas a jubilar en Chile (SENAMA, 2016), el cual demuestra cómo a partir de los 75 años y especialmente en la población femenina decrece significativamente la presencia de este grupo dentro del mercado laboral y empeoran las condiciones laborales de quienes se encuentran empleados. Según los datos de esta encuesta apenas el 12\% realiza labor remunerada al menos una vez a la semana.

Es interesante observar el dato entre las actividades relacionadas con cocinar y labores domésticas y la realización de compras, al interior de las comunas. En Las Condes alcanza un 94\% las compras, mientras un 77\% sostiene que cocina y/o realiza labores domésticas, mientras en Recoleta el 94\% realiza como actividad más frecuente la relacionada a las labores domésticas mientras un $84 \%$ se indica realiza compras al menos una vez a la semana. Las desigualdades económicas (de ingresos) sin dudas median el tipo de labor que realizan con más frecuencia, pero sí resalta en ambos casos el bajo nivel de presencia en el mercado laboral, esto debido a la condición de vejez. Solo el 12\% del total realiza labores remuneradas. En Recoleta el 13\% y en Las Condes el 11\%. Este dato expresa cómo si bien otras variables como nivel educacional, ingresos, dan cuenta de una desigualdad de clase, la condición vejez unifica y re-enclasa ${ }^{3}$ a este grupo en una nueva matriz de producción de desigualdad persistente.

Se consultó por la realización al menos una vez a la semana de las siguientes actividades: (1) labores remuneradas, (2) cuidado de otros familiares, (3) compras, (4) cocinar u otras labores domésticas.

Siguiendo la idea de Bourdieu del enclasamiento que producen los agentes dentro del campo, así como de los objetos y prácticas en tanto enclasables. Refiere a las operaciones de enclasamiento en el sentido de distinción social y posicionamiento dentro del campo (Bourdieu, 1998, pp. 169-171) 
Nivel de dependencia en la administración de bienes materiales propios

Un importante indicador respecto del cual se midieron los niveles de dependencia en el ámbito económico fue la capacidad en la administración de bienes materiales propios. Respecto a la pregunta ¿administra usted su dinero y bienes materiales?, solo el 34\% declaró que sí, el 33\% que no, y otro 33\% con ayuda de otra persona, mostrando una fragilidad en la gestión y autonomía de su patrimonio. La dependencia no es expresión de abuso patrimonial pero sí constituye una condición que favorece o potencia aquella, entre otras expresiones de maltrato como los psicológicos. En este sentido que el 66\% de estas personas mayores, en un rango de 75 años no administren sus bienes materiales constituye sin dudas un rasgo de dependencia que dice de condiciones de desigualdad de poder en las cuales son insertos los adultos mayores, al ser percibidos y tratados como sujetos dependientes, aún cuando no lo sean según sus capacidades físicas y cognitivas. Si recordamos que se trata fundamentalmente de mujeres, podemos sostener que hay un claro marcador de desigualdad que se reproduce en lo referido a la posición de estas en relación a la propiedad, en este caso a su administración, a partir de una sub-representación.

El hecho de que la muestra estudiada indique se trata de personas mayores que aportan más recursos a la familia que lo que reciben de esta, con una presencia significativa en la producción de trabajo doméstico no remunerado, con baja autonomía en la administración de sus bienes y que sea una muestra de mujeres mayoritariamente, revela la presencia de un marcador de desigualdad donde el género también media fuertemente las experiencias de envejecimiento en los grupos estudiados.

Otra variable influyente en los niveles de dependencia económica fue el nivel educacional. La capacidad de administración del patrimonio se concentró en aquellos grupos con mayor nivel educacional. Las personas que tenían nivel universitario (30,5\%) y enseñanza media $(37,1 \%)$ eran los que sí administran sus bienes y dinero. Así mismo de las personas que no administran sus bienes o lo realizan con ayuda de otra persona, el $27 \%$ ha sentido temor de ser engañado por esa persona, porcentaje que incrementa en Las Condes a un $33 \%$. Este dato no es menor, representa que al menos 1 de cada 4 personas de quienes han traspasado o compartido la administración de sus bienes con otros, sienten que pueden sufrir abuso patrimonial. Las cifras reflejan las intersecciones entre desigualdades múltiples (educación, ingresos) y la producción de dependencia como condicionamiento favorable para experiencias de abuso patrimonial y la perpetuación de la desigualdad en el caso de las personas mayores.

\section{Experiencias de abuso patrimonial}

Respecto de experiencias directas de abuso patrimonial un 16\% declaró haber sido estafado, robado o engañado aprovechándose de su condición de adulto mayor. En el caso 
de Recoleta, donde como hemos visto también se confirma con un nivel educacional inferior al de Las Condes, este porcentaje sube al 19\% de las personas abusadas de su patrimonio.

En general respecto a las condiciones de dependencia y expresiones de abuso patrimonial, los resultados muestran un grupo de adultos mayores que aporta más económicamente a la familia que las ayudas recibidas. Es la familia el principal pilar proveedor de ayudas económicas, las que en general y para cada caso en particular son inferiores a las entregadas por los adultos mayores, en su mayoría mujeres. Se expresa una clara asociación entre el nivel educacional, el monto de las pensiones, el género y la capacidad de autonomía respecto de la administración de su patrimonio. Si bien los casos de abuso patrimonial declarados son minoría, sí se evidencia cómo estos van asociados al nivel educacional de las personas mayores. Así mismo las condiciones de fuertes apoyos económicos provenientes del propio grupo familiar, podrían potenciar la existencia de abuso patrimonial, quedando en el plano privado y de las relaciones afectivas, situaciones de abuso que pueden estar siendo naturalizadas, invisibilizadas u ocultadas intencionalmente. A su vez la relativa poca autonomía respecto de su patrimonio, el cual solo el 33\% administra autónomamente, potencia también la presencia de expresiones de abuso patrimonial asociadas a la condición de género, además.

El mayor contraste encontrado en esta dimensión radica en la alta capacidad productiva de las personas mayores frente a los bajos niveles de independencia económica en la gestión de su propio patrimonio y el acceso a fuentes de ingresos cuyo origen sea directamente trabajo remunerado. Todo ello enciende alarmas respecto a situaciones de abuso patrimonial no reconocidas como tal que afectan el bienestar de las personas mayores. Y a su vez confirman la presencia de una desigualdad producida bajo la condición estructurada entorno a la vejez.

\section{Maltrato Psicológico}

De las formas de maltrato indagadas, el maltrato psicológico resalta como el más presente entre las experiencias de las personas mayores. El 66\% de los encuestados/as afirmó haber vivido alguna experiencia de maltrato psicológico en el último año. De ellos el $31 \%$ de los/as encuestados/as declaran haberse sentido o sentirse solos durante el último año. La sensación de soledad no es un indicador de experiencias de maltrato directamente, pero llama la atención y puede remitir situaciones de maltrato u abandono cuando este se presenta en contextos de adultos mayores que tienen hijos y/o conviven con familiares. El 87\% de los/as encuestados/as tiene hijos y el 71\% vive con familiares, aún así el 31\% declara sentirse solos/as.

El sentimiento de soledad puede ser expresión directa del aislamiento, incluso cuando como en la mayoría de los/as encuestados se convive con otras personas, fundamentalmente familiares. El aislamiento además de ser expresión de maltrato psicológico, constituye una de las razones por las cuales las personas invisibilizan u ocultan la presencia de maltratos, generando un círculo vicioso: el miedo a la soledad o el aislamiento. 
Llama la atención que junto con el sentimiento de soledad, la segunda experiencia de maltrato más presente fue el ser ignorado por otros: "Cuando pregunto algo no me responden". Un 24\% declara que en el último año al menos una vez cuando pregunta algo no le responden. Esta expresión de maltrato va muy aparejada al aislamiento y la producción de sentimiento de soledad hacia las personas mayores. La exclusión a la que son sometidos incluso dentro del propio grupo familiar son expresiones de maltrato, invisivilizadas y naturalizadas pero al parecer altamente frecuentes.

También resaltó el hecho de haberse sentido intimidados por personas más jóvenes (20\%), la presencia de gritos (19\%) y humillaciones (12\%). El obtener estos datos sobre una materia de difícil y doloroso reconocimiento apunta a una presencia considerable de expresiones de maltrato psicológico del cual son víctimas las personas mayores y fundamentalmente desde el espacio familiar.

\section{¿En el último año ha tenido alguna de estas sensaciones o experiencias?}

TOTAL

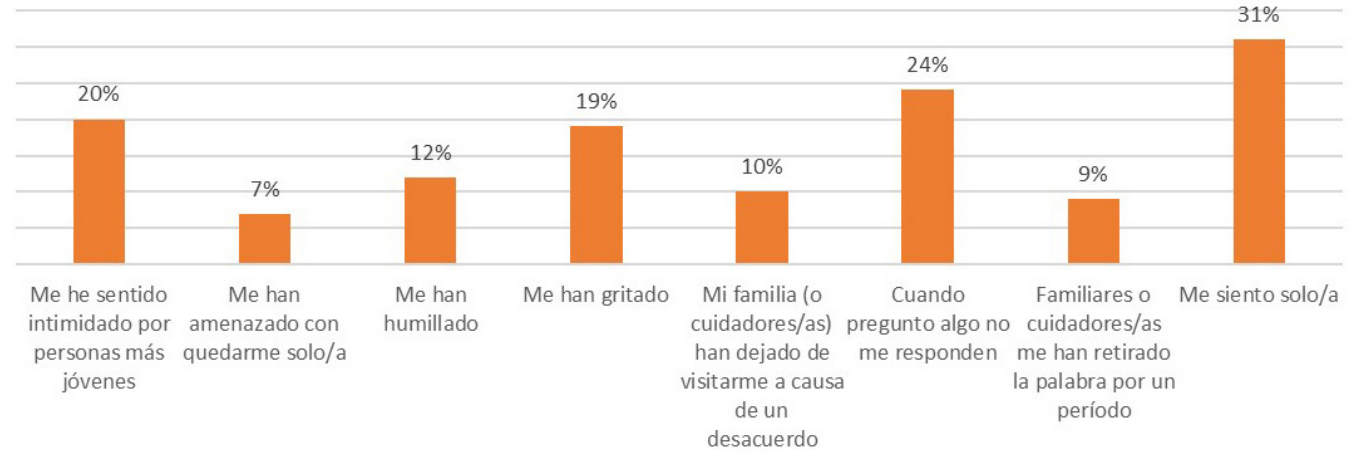

Fig. 1. Expresiones del maltrato psicológico. Total Elaboración propia. EPTAM, 2017.

La distribución de este tipo de maltrato al interior de ambas comunas no presenta diferencias notables, lo cual confirma cómo el maltrato psicológico está asociado más que a la clase, a la condición de ser adultos mayores, percibidos y/o tratados como sujetos dependientes o con menos valor. Los datos confirman la presencia de una desigualdad persistente en el trato que reciben estas personas por su sola condición de ser personas mayores.

Otro dato interesante es que del porcentaje de personas mayores que han tenido alguna experiencia de maltrato psicológico, el 45,5 \% de ellos conviven con familiares. Por el contrario, quienes conviven con otras personas no familiares apenas declaran en un $2 \%$ el haber tenido alguna experiencia de maltrato psicológico, mientras el 19\% de quienes viven solos han vivenciado alguna expresión de maltrato psicológico.

La confluencia de experiencias de maltrato psicológico, donde el aislamiento y la soledad se muestran como las formas más recurrentes, y el hecho de que estas se produzcan 
¿En el último año ha tenido alguna de estas sensaciones o

experiencias?

Recoleta

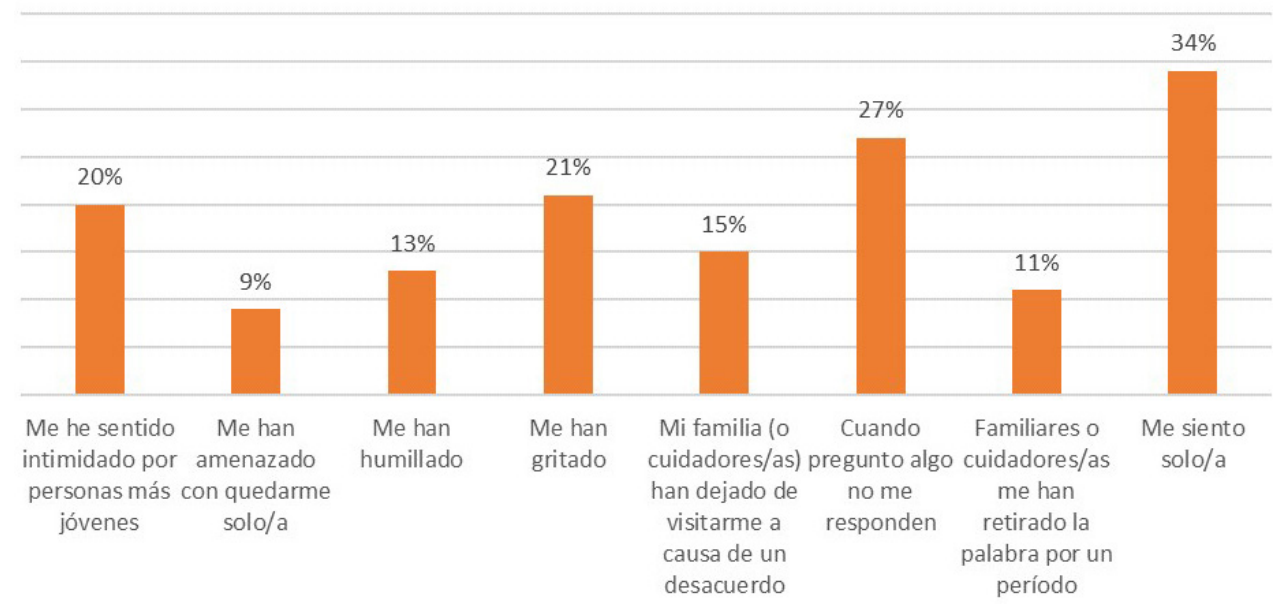

Fig 2 Expresiones de maltrato psicológico. Recoleta.

Elaboración propia. EPTAM, 2017.

¿En el último año ha tenido alguna de estas sensaciones o

experiencias? Las Condes

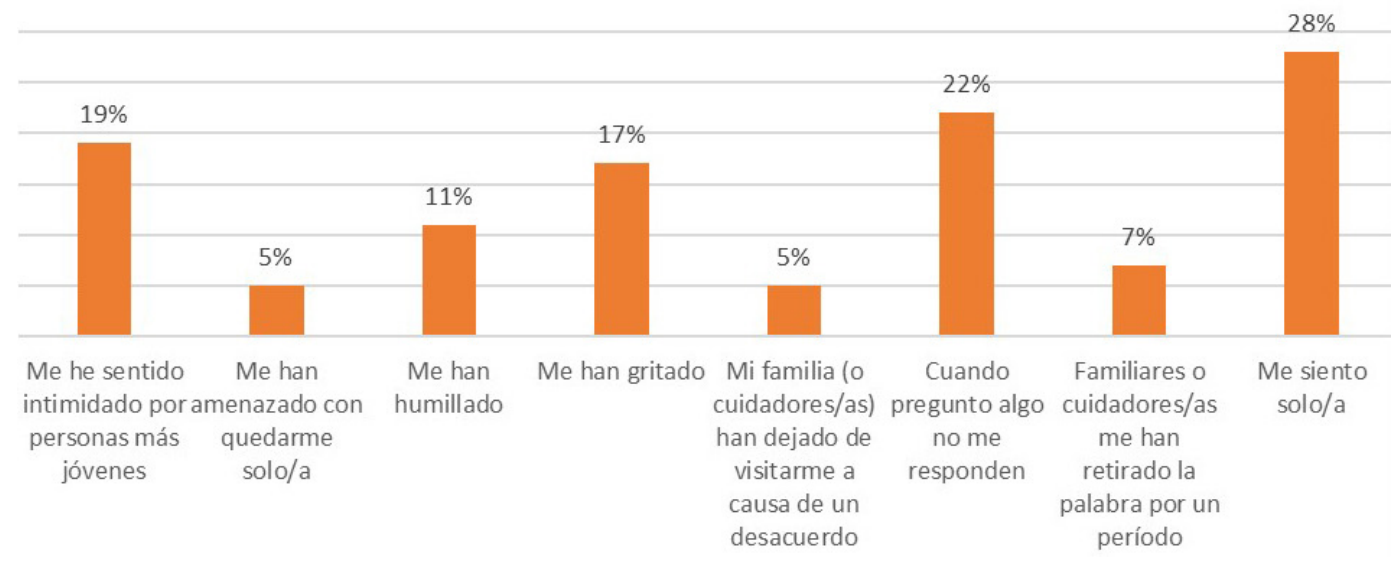

Fig 3 Expresiones de maltrato psicológico. Las Condes

Elaboración propia. EPTAM, 2017.

mayoritariamente en contexto de convivencia de las personas mayores con sus familiares, vuelve más alarmante el dato y reafirma estudios anteriores que sostienen que los maltratos hacia las personas mayores tienen como origen principal los cuidadores/as- familiares (Del Pozo, 2014; Briceño; et.al: 2014; Zegers, 2014).

Igualmente alarmante resulta que siendo que el $71 \%$ de los/as encuestados/as vive con familiares, y que el 62\% realiza aportes monetarios al grupo familiar, exista un 66\% que declara haber vivido alguna experiencia de maltrato psicológico. Así como existe una directa 
relación entre el maltrato psicológico y los aportes monetarios realizados por los adultos mayores a sus familias, también se expresa un vínculo directo entre quienes tienen hijos y han vivido alguna expresión de maltrato psicológico. Más del 57\% de los/as encuestados/as que tienen hijos han experimentado algún tipo de maltrato psicológico.

\section{Nivel de dependencia física e intelectual}

Los niveles de dependencia física e intelectual de la muestra estudiada fueron muy bajos. Como se planteó en las consideraciones metodológicas, estas personas se caracterizaron por ser una población con niveles de empoderamiento medio-elevado y que buscaban reforzar dicho empoderamiento mediante las actividades que desarrollaban en los círculos y lugares de encuentro organizados desde las municipalidades y los programas de SENAMA. El porcentaje más alto es el referido a los apoyos requeridos para la realización de trámites (8\%) en ambas comunas. Este porciento sube a 11\% en la comuna de Recoleta, sin olvidar la posible influencia del nivel educacional que es inferior respecto a Las Condes.

Dentro de estos bajos niveles de dependencia respecto de instituciones, resalta la familia como aquella de la cual se sienten más dependientes (29\%). Lo cual se condice con el hecho de ser la familia el principal sector proveedor de cuidados a los adultos mayores. En el caso de Las Condes un 37\% se dice sentirse dependiente de la familia, un 18\% frente al Estado y un 11\% respecto del Mercado. En cambio en Recoleta el 31\% reconoce tiene alguna dependencia respecto del Estado, un 20\% de la familia y un 9\% del Mercado. El contraste de las cifras entre las comunas respecto de la depenendencia Estado/Familia, da cuenta de un lazo más fuerte entre Estado y adultos mayores en Recoleta, siendo incluso superior en cuanto a dependencia de la Familia, mientras en Las Condes la percepeción de dependencia del Estado es menor y prevalece la dependencia respecto de la Familia. La configuración de clase que se expresa en esta dos comunas, así como los diferentes modelos de gestión en el ámbito de políticas públicas de los gobiernos municipales marcan claramente este resultado.

Pareciera que en aquellos espacios más privatizados la familia termina siendo la contenedora de los adultos mayores, frente a otros donde el fortalecimiento o mayor presencia de políticas públicas implican una descompresión del núcelo familia como principal proveedor de cuidados. Conociendo además que es en el ámbito familiar donde se producen la mayor cantidad de maltratos hacia los adultos mayores, más cuando son dependientes de cuidadores familiares, entonces potenciar el vínculo entre Estado y adultos mayores es una urgencia no solo para el bienestar de las personas mayores sino también de las familias y la sociedad en general. El camino efectivo para contrarrestar la desigualdad no es y no ha sido nunca un camino individual, sino que amerita una gestión que articule los tres actores fundamentales en la constitución de todo régimen de bienestar: Estado, Familia, Estado (Martínez, 2008; Esping-Andersen, 1993). 


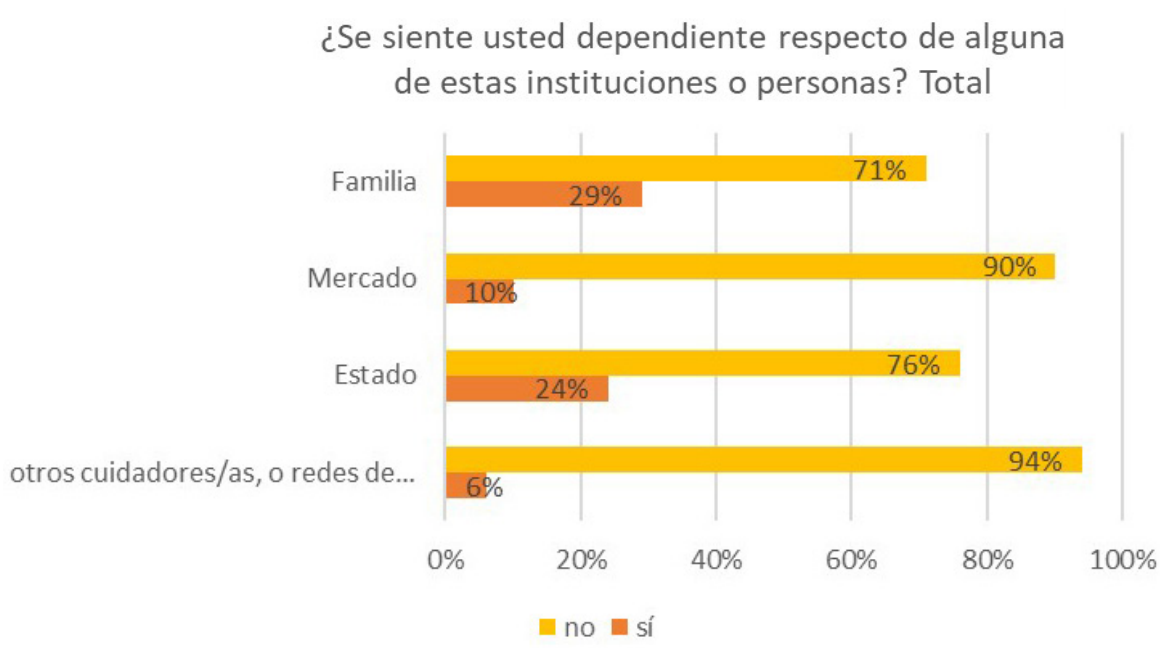

Fig 4. Instituciones de las que se sienten dependientes las personas mayores. Total Elaboración propia. EPTAM, 2017.

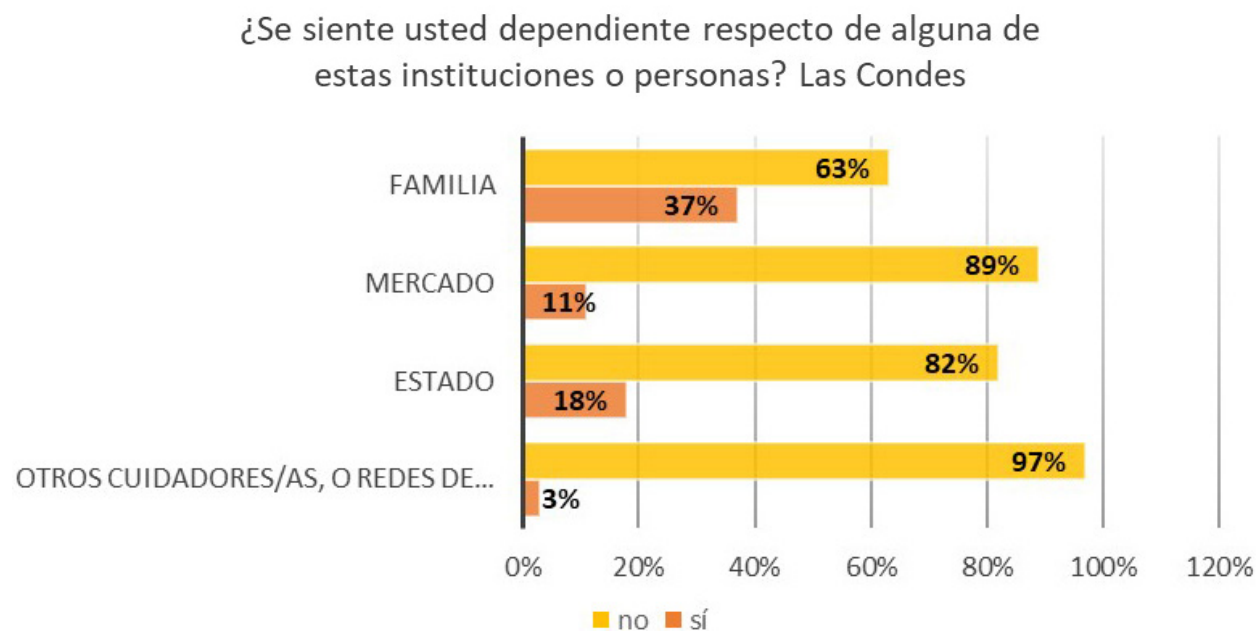

Fig 5 Instituciones de las que se sienten dependientes las personas mayores. Las Condes Elaboración propia. EPTAM, 2017.

Derechos de las personas mayores

El ámbito de los derechos provee no solo un enfoque desde donde interpelar la realidad de las personas mayores, sino que es un espacio en sí desde donde se fortalece o debilita el bienestar de los adultos mayores, desde donde también se potencia o mitiga la producción de desigualdades. La encuesta indagó en torno al conocimiento que tienen los adultos mayores respecto de sus derechos y su percepción sobre los mismos. La existencia, fortalecimiento, conocimiento y uso de derechos específicos para los/as adultos mayores constituye una herramienta de enfrentamiento y por lo tanto de reconocimiento de desigualdades originadas desde esta condición. El robustecimiento de los derechos humanos de las personas mayores, 


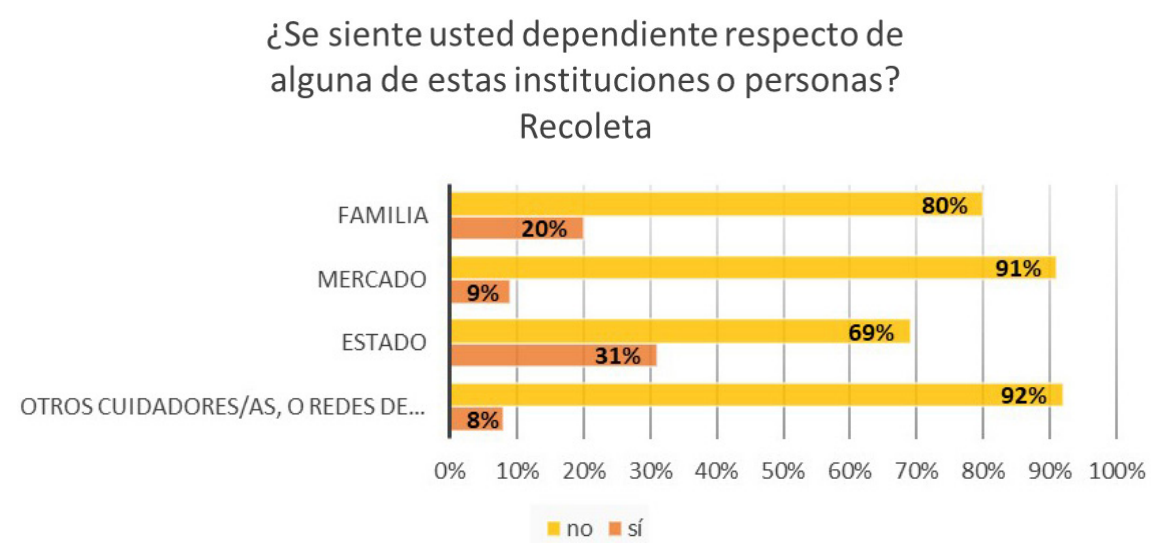

Fig 6. Instituciones de las que se sienten dependientes las personas mayores. Recoleta Elaboración propia. EPTAM, 2017.

es en sí un mecanismo indispensable en la lucha contra el maltrato como forma de expresión de la desigualdad.

La reciente firma por parte de Chile de la Convención Interamericana sobre la protección de los Derechos Humanos de las Personas Mayores en 2016 fortalece un camino en tal sentido, sin embargo según las percepciones de los/as encuestados/as en materia de sus derechos, estos son aún muy insuficiente. Con lo cual se evidencia la escasa protección (al menos percibida como tal) frente a condiciones de desigualdad categorial que viven y de la cual dan cuenta los resultados de la EPTAM.

Frente a la pregunta: ¿cree usted que los derechos de los adultos mayores en Chile son vulnerables?, un 84\% consideró que sí lo son. Los ámbitos donde se expresaron mayormente la vulneración de sus derechos fueron los de la salud pública con un 63\%; las AFP con un 53\%; el Mercado Laboral con 53\% y la Familia con un 49\%, seguidos por el Estado con un 40\%. Este orden representa una clara disposición de necesidades de cuidados, recursos, accesos que se concentran en el derecho a la salud, pensión digna, trabajo y cuidados en todo su sentido, físico y emocional, los cuales se vulneran según la percepción de los/as encuestados/as. La importancia y centralidad de estos ámbitos donde debieran resguardarse y fortalecerse los derechos de las personas mayores resultan ser aquellos donde según su percepción se producen las mayores vulneraciones.

La percepción del trato recibido por distintas instituciones también fue un indicador mediante el cual se exploró dónde se vulneran con mayor frecuencia los derechos de este grupo y en consecuencia desde dónde se reproducen principalmente las desigualdades. En este sentido el 32 \% de las personas declaran haber recibido un trato injusto en el transporte público, 24\% sienten que las personas jóvenes los tratan injustamente, mientras un 23\% refiere estos tratos injustos han provenido de los servicios de salud pública. En el caso de la Salud Pública en Recoleta el porciento alcanza un 30\%, a diferencia de Las Condes donde solo el 12\% declara haber sido tratado injustamente en estos servicios. La diferencia está marcada por los usos que hacen cada uno de estos grupos de los servicios e instituciones 
indagadas. El nivel socioeconómico de los/as adultos mayores de Las Condes, aún con su heterogeneidad es claramente superior al de los/as de Recoleta, lo cual indica el poco uso que los primeros hacen del transporte público o los servicios de salud pública por ejemplo. En general este es un grupo cuyas demandas y necesidades son resueltas en espacios privados, ya sea la familia o el mercado. Mientras las personas de Recoleta hacen un uso mucho más intensivo del Estado y sus programas. En ese sentido son mucho más dependientes de él, como usuarios activos, y por lo mismo también más críticos de los servicios recibidos. Tal es así que en los indicadores de servicios públicos cuestionados (salud, transporte, otros servicios municipales/públicos), los/as encuestados de Recoleta doblan en porcentaje las respuestas de Las Condes que afirman haber recibido un trato injusto en estas.

Sin embargo, más allá de estas diferencias, atravesadas por las desigualdades de clase, hay un elemento común y es que en ambas comunas después del transporte público, quienes sienten más los tratan injustamente son las personas jóvenes, en Recoleta lo considera un 29\% y en Las Condes un 20\%. El par categoríal joven/viejo se refuerza como ente desde donde se reproduce la desigualdad persistente respecto de quienes ya no son jóvenes.

\section{CONCLUSIONES}

Los resultados presentados hasta acá permiten concluir la existencia de un conjunto de desigualdades que atraviesan el envejecimiento de la muestra estudiada. El nivel educacional, asociado a su vez a los recursos económicos que se perciben (monto pensiones), marcan rasgos específicos respecto de los vínculos y tipos de dependencias de los/as adultos mayores en relación a determinados actores e instituciones sociales. Resalta una mayor dependencia del Estado allí donde los sujetos se encuentran en situación de mayor vulnerabilidad socioeconómica; mientras en quienes poseen mayores recursos económicos y educacionalesculturales, apenas refieren un vínculo con el Estado y concentran su dependencia en la familia como principal proveedora de cuidados.

Ante la pregunta, ¿el envejecimiento en Chile está atravesado por diferencias de clase?, los resultados nos permiten fundamentar que las diferencias encontradas entre los adultos mayores estudiados en Recoleta y Las Condes dan cuenta de condiciones materiales e intelectuales fundamentalmente (ingresos, nivel educacional) desiguales entre unos y otros. A su vez el nivel educacional y los ingresos son dos variables intervinientes en los niveles y tipos de dependencia, principalmente de tipo económica. Los niveles de dependencia económica en lo referido a la administración autónoma de su patrimonio de modo puntual, marcan estas desigualdades, obteniendo una mayor autonomía en la gestión patrimonial aquellos sujetos con mayor índice educacional. Por otro lado, considerando que más del 70\% de la muestra eran mujeres, se refuerza el vínculo entre desigualdad de género y patrimonio, reafirmando el hecho de una sub-representación de las mujeres en materia de propiedad ampliamente estudiados en América Latina (Deere y León, 2002). 
Sin embrago a pesar de que solo el 33\% declara tener autonomía sobre la administración de sus bienes e ingresos, no podemos sostener se tratan de adultos mayores totalmente dependientes económicamente, puesto que el $62 \%$ realiza aportes monetarios al grupo familiar, siendo estos aportes superiores en aquellos grupos con mayores ingresos. Es significativo que por sobre las diferencias en cuanto a ingresos de los grupos estudiados, más de la mitad realiza aportes monetarios al grupo familiar. Así mismo si bien han quedado fuera del mercado laboral en su mayoría, puesto que solo el 12\% realiza labores remuneradas, el $85 \%$ se encarga al menos una vez a la semana de las labores domésticas del hogar. La invisibilización del trabajo de cuidados, como trabajo, se reproduce también porque el mismo se distribuye desigualmente a partir de una organización categorial-desigual: hombre-mujer, joven-viejo. Este es un punto central donde se demuestra que la producción de la desigualdad persistente a partir de la categoría "viejo", termina siendo capitalizada por quienes reproducen un sistema de organización y acceso a recursos excluyente.

La minusvaloración de los adultos mayores, la percepción de estos como sujetos dependientes, menos capaces (Guajardo, 2013; FACSO/FLACSO Chile/ SENAMA, 2013) hace, como en el caso de las mujeres, que se les ubique en una situación de vulnerabilidad donde la explotación sobre ellos, su trabajo, y hasta su cuerpo, sea mayor y "justificada”. Claro está cuando se juntan ambas condiciones, ser adulto mayor y mujer, se potencian doblemente estas explotaciones y la producción de desigualdad persistente se amplifica así como las condiciones de pauperización de estas sujetos.

Los mecanismos de exclusión, control y cierre social de los que habla Tilly (2000) los encontramos tanto en su baja inserción en el mercado laboral como en la presencia de maltratos, fundamentalmente psicológicos a los que son sometidas las personas mayores, más allá de su condición de clase.

En este sentido la afirmación sobre la mediación de la desigualdad de clase como un rasgo del envejecimiento en Chile, hay que complejizarla un tanto más. Podemos concluir a partir de los resultados presentados que la sola condición del envejecimiento hace que se compartan condiciones de desigualdad estructural comunes para los adultos mayores más allá de sus diferencias de clase en la muestra estudiada. En este sentido el envejecimiento es usado precisamente como categorial basal en la producción de desigualdad persistente. Los altos índices de maltrato psicológico dan cuenta de ello, en ambas comunas, y este maltrato es más elevado en aquellos casos donde los adultos mayores conviven con familiares. Del total de las personas que han sido víctimas de alguna experiencia de maltrato psicológico, el $45,5 \%$ de ellos conviven con familiares. Igualmente contrastante resulta que de las personas que han sufrido maltrato psicológico en el último año el 41,4\% de ellos/as realizan aportes económicos al grupo familiar, subiendo al $45 \%$ en Las Condes. Por otra parte más del 57\% de los/as encuestados/as que tienen hijos han experimentado algún tipo de maltrato psicológico.

Los vínculos entre las experiencias de maltrato psicológico y los aportes monetarios realizados por los adultos mayores a sus familias, la presencia de hijos y la convivencia con 
otros familiares expresan un claro mecanismo de operación de la desigualdad persistente que como toda desigualdad es favorable a quienes controlan y tienen el poder categorial.

Uno de los resultados principales de la EPTAM permite concluir que la presencia de maltrato estructural es la base potencial de otras expresiones del maltrato para este grupo. Los derechos referidos a las personas mayores constituyen una base fundamental en el resguardo de un bienestar garantizado a ellas. La elevada percepción respecto a la vulneración de esos derechos en Chile y a la vez el poco conocimiento respecto de los mismos denota un terreno donde aún queda mucho por avanzar, no solo en términos jurídicos-normativos, sino también culturales y redistributivos.

Es la reproducción de las desigualdades también una responsabilidad societal y política, y es ella una condicionante de las situaciones de maltrato, por ejemplo, las expresadas en términos del género y de la vejez misma. Solo el camino de una política hacia la vejez y el envejecimiento que tenga en su base el reconocimiento y también la redistribución (Fraser, 2006), podrán proveer condiciones de justicia social para las personas mayores. Ahora bien, es imprescindible también pensar críticamente los marcos de dicho sistema de redistribución, en tanto no se les dé continuidad a pares categoriales productores de desigualdad persistente, como el caso de joven-viejo, hombre-mujer. En otros términos, se requiere un marco redistributivo que rompa con los pares categoriales productores y legitimadores de desigualdad persistente en la sociedad. El verdadero reconocimiento de las personas mayores, sus derechos, pasa necesariamente por condiciones redistributivas que minimicen la reproducción de las desigualdades. La sola presencia de estas, las cuales se agudizan en situaciones de vulnerabilidad como la vejez - y aún más, son ellas las que hacen de la vejez una situación de vulnerabilidad-, implican un maltrato estructural hacia las personas mayores que como sociedad reproducimos. La desigualdad persistente produce maltrato estructural. La lucha contra el maltrato debe ser antes bien pensada como una lucha contra la desigualdad categorial.

\section{REFERENCIAS}

Abusleme, M., \& Caballero, M. (2014). El maltrato hacia las personas mayores: realidad y desafíos del abordaje desde las políticas públicas en Chile a través del SENAMA. En M. Abusleme \& M. Caballero (Ed.), Maltrato a las personas mayores en Chile. Haciendo visible lo invisible (pp. 9-18). Santiago de Chile: Ediciones SENAMA.

Bourdieu, P. (1998). La distinción. Criterios y bases sociales del gusto. Buenos Aires: Taurus.

Briceño, C., Navarrete, G., \& Carrasco, V. (2014). Cuidadores y maltrato en la persona mayor: estrategias para su abordaje. En M. Abusleme \& M. Caballero (Eds.), Maltrato a las personas mayores en Chile. Haciendo visible lo invisible (pp. 155-170). Santiago: Ediciones SENAMA.

Caballero, M., \& Massad, C. (2013). Antecedentes en torno al maltrato hacia las personas 
mayores en Chile En M. Abusleme \& G. Guajardo (Ed.), El maltrato hacia las personas mayores en la Región Metropolitana, Chile. Investigación cualitativa en vejez y envejecimiento (pp. 21-32). Santiago de Chile: Ediciones SENAMA. CASEN. Encuesta Una Medición de la Pobreza Moderna y Transparente para Chile. Recuperado de: http:// observatorio.ministeriodesarrollosocial.gob.cl/documentos/Presentacion_Resultados_ Encuesta_Casen_2013.pdf

CEPAL (2009). El envejecimiento y las personas de edad. Indicadores sociodemográficos para América Latina y el Caribe. Santiago de Chile: CEPAL.

Del Pozo, M. (2014). Familia y soportes intergeneracionales en la adultez mayor: riesgos y desafíos. En M. Abusleme \& M. Caballero (Ed.), Maltrato a las personas mayores en Chile. Haciendo visible lo invisible (pp. 127-136) . Santiago de Chile: Ediciones SENAMA.

Deere, C., \& León, M. (2002). Género, propiedad y empoderamiento: tierra, Estado y Mercado en América Latina. México: UNAM.

Escuela Sociología. Universidad Católica Silva Henríquez. (2017). Encuesta Percepción del Trato hacia Adultos Mayores. (inédito)

Esping-Andersen, G. (1993). Los tres mundos del Estado del Bienestar. Valencia: Edicions Alfons El Magnanim.

Fraser, N., \& Honneth, A. (2006). ¿Redistribución o reconocimiento? Madrid: Ed Morata.

González, S. (2014) Envejecimiento, subjetividad y maltrato. En M. T. Abusleme \& M. Caballero (Eds.), Maltrato a las personas mayores en Chile. Haciendo visible lo invisible (pp. 119126). Santiago de Chile: Ediciones SENAMA.

Gonzálvez, H. (Ed.). (2015). Diversidades familiares, cuidados y migración. Nuevos enfoques y viejos dilemas. Santiago de Chile: Ediciones UAH.

Gonzálvez, H. (2017). Ser mujer en Santiago de Chile: feminización de los cuidados en la vejez y desigualdades acumuladas. En A. Vera (Ed.), Malestar social y desigualdades en Chile (pp. 173-194). Santiago de Chile: Ediciones UAH.

Guajardo, G. (2013). Una tipología de las significaciones y sentidos sociales sobre el maltrato desde la mirada de las personas mayores. En M. T. Abusleme \& G. Guajardo (Ed.), El maltrato hacia las personas mayores en la Región Metropolitana, Chile (pp. 127-138). Santiago de Chile: Ediciones SENAMA- Flacso.

Huenchuan, S., González, D., Paredes, M., \& Guzmán, J. (2007). Protección y participación en la vejez: escenarios futuros y políticas públicas para enfrentar el envejecimiento en Chile. Santiago: CEPAL/CELADE.

Huenchuán, S. (Ed.), (2009). Envejecimiento, derechos humanos y políticas públicas. Santiago de Chile: CEPAL.

Huenchuán, S. (2014). El maltrato hacia las personas mayores: conceptos, normas y experiencias de políticas en el ámbito internacional. En M.T. Abusleme \& M. Caballero (Eds.), Maltrato a las personas mayores en Chile. Haciendo visible lo invisible. Santiago de Chile: Ediciones SENAMA. 
Martínez Franzoni, J. (2008). Domesticar la incertidumbre en América Latina. Mercado Laboral, política social y familias. Costa Rica: UCR.

Mora, T. (2013). Avances normativos en Chile respecto del maltrato a las personas mayores. En G. Guajardo \& M. T. Abusleme (Ed.), El maltrato hacia las personas mayores en la región metropolitana, Chile. Investigación cualitativa en vejez y envejecimiento (pp.3353). Santiago de Chile: Ediciones SENAMA.

SENAMA. (2005). Definición y Tipificación del Maltrato al adulto mayor en Chile. Documento de trabajo. Recuperado de: http://www.senama.cl/filesapp/1288.pdf

SENAMA. (2009). Las personas mayores en chile. Situación, avances y desafíos del envejecimiento $y$ la vejez. Santiago de Chile: SENAMA.

SENAMA/FACSO/FLACSO Chile. (2013). Encuesta Inclusión y exclusión social de las personas mayores en Chile. Opiniones, percepciones, expectativas y evaluaciones. Tercera encuesta nacional de inclusión y exclusión social. FACSO, FLACSO Chile, SENAMA.

SENAMA. (2016). Informe Mercado Laboral, Adulto Mayor y Personas próximas a jubilar en Chile. Santiago de Chile: SENAMA.

Tilly, Ch. (2000). La desigualdad persistente. Argentina: Manantial.

Thumala, D. (2011). Formas de afrontamiento a las pérdidas asociadas al envejecer. Tesis en opción al grado de Doctor en Psicología. UPC.

Valenzuela, R. (2016). Informe Mercado Laboral, Adulto Mayor y Personas próximas a jubilar en Chile. Santiago de Chile: SENAMA.

Zegers, B. (2014). El maltrato a las mujeres adultas mayores. En M. T. Abusleme \& M. Caballero (Eds.), Maltrato a las personas mayores en Chile. Haciendo visible lo invisible (pp.137154). Santiago de Chile: Ediciones SENAMA. 